elSSN: 2444-7986

DOI http://dx.doi.org/10.14201/orl201671.13531

Artículo de revisión

\title{
ACTUALIZACIÓN DEL TRATAMIENTO DE LA FARINGOAMIGDALITIS RECURRENTE DEL ADULTO. REVISIÓN
}

\section{Updating the treatment of recurrent tonsillitis in adults. Review}

\author{
María Cristina GASCÓN-RUBIO*; Albino José ALONSO-ALONSO \\ SACYL. Hospital Santiago Apóstol. Servicio de Otorrinolaringología. Miranda de Ebro. Burgos. España. \\ *Correspondencia: mcgasconr@saludcastillayleon.es
}

Fecha de recepción: 31 de octubre de 2015

Fecha de aceptación: 20 de diciembre de 2015

Fecha de Publicación: 15 de enero de 2016

Conflicto de intereses: Los autores declaran no tener conflictos de intereses

Imágenes: Los autores declaran haber obtenido las imágenes con el permiso de los pacientes

Política de derechos y autoarchivo: se permite el autoarchivo de la versión post-print (SHERPA/RoMEO)

Licencia CC BY-NC-ND. Licencia Creative Commons Atribución-NoComercial-SinDerivar 4.0 Internacional

(c) Universidad de Salamanca. Sucomercialización está sujeta al permiso del editor

RESUMEN

\begin{abstract}
Introducción y Objetivos: La faringoamigdalitis aguda en el adulto (FAA) es uno de los motivos de consulta más frecuente en Atención Primaria. El objetivo de esta revisión es actualizar las opciones terapéuticas existentes para la prevención y tratamiento de las FAA y determinar que intervenciones tienen mayor impacto en la disminución de la morbilidad y mejora de la calidad de vida de los pacientes, lo cual incide directamente en el consumo de recursos sociosanitarios. Material y Método: Se definieron los criterios clínicos de la FAA, las indicaciones de amigdalectomía y la pautas de antibioterapia según las guías clínicas de las sociedades científicas nacionales e internacionales. Se revisó la literatura relacionada con el tratamiento de la FAA incluyendo otras opciones terapéuticas no contempladas en las guías clínicas previas (extracto de P. Leucotomos, vacunación por la vía de las mucosas, AM3 y betaglucanos, homeopatía y fitoterapia) en las bases de datos ClinicalKey y PubMed. Resultados: La comparación de los estudios resultó dificultosa debido a la disparidad de criterios de inclusión y diagnósticos, tamaños muestrales, tiempo de seguimiento y la escasa uniformidad en las escalas que miden las variaciones clínicas. Conclusiones: No podemos concluir si una opción terapéutica es más efectiva que otra en el tratamiento y prevención de las FAA del adulto.
\end{abstract}

PALABRAS CLAVE Faringitis; Amigdalitis; Amigdalectomía; Enfermedades Otorrinolaringológicas; Terapias complementarias.

SUMMARY

Introduction and Objectives: Acute pharyngitis in adults (APA) is one of the most frequent reasons for consultation in primary care. The main objective of this review is to update the therapeutic options for the prevention and treatment of) APA and to determine which interventions have the greatest impact in reducing morbidity and improving the quality of life of patients, which directly affects the consumption of health resources. Material and Methods: First clinical criteria of the APA, indications of tonsillectomy and patterns of antibiotic therapy 
according to clinical guidelines of national and international scientific societies were defined. Subsequently, the literature related to the treatment of the APA including other therapeutic options not covered in previous clinical guidelines (extract P. Leucotomos, vaccination by the mucosal route, AM3 and beta-glucans, homeopathy and herbal medicine) was revised. ClinicalKey bases and PubMed data were used. Results: The comparison of the studies proved difficult due to the disparity of criteria for inclusion and diagnostics, sample sizes, time tracking and poor uniformity in the clinical scales measuring variations. Conclusions: Therefore we can not conclude whether a therapeutic option is more effective than another in the treatment and prevention of adult APA.

KEYWORDS

Pharyngitis; Tonsillitis; Tonsillectomy; ENT diseases; Complementary Therapies.

\section{INTRODUCCIÓN}

La faringoamigdalitis aguda en el adulto (FAA) es uno de los principales motivos de consulta en Atención Primaria (AP) e incluso de los servicios de urgencias. Su etiología más frecuente es viral. Entre las principales causas bacterianas destaca el Streptococcus Pyogenes o estreptococo $B$-hemolítico grupo A (EBHGA) que puede llegar a ser el agente causal (según series) de hasta en el $30 \%$ de los casos en adultos jóvenes y muy poco frecuente en mayores de 50 años [1].

Se definen como amigdalitis de repetición [2] o amigdalitis recurrentes las siguientes situaciones clínicas:

- 7 o más episodios de amigdalitis aguda al año en el último año o,

- 5 episodios al año en los últimos dos años 0 ,

- 3 episodios al año en los últimos 3 años.

- Síntomas persistentes durante al menos 1 año.

La FAA representa una causa de absentismo laboral de hasta 6,5 días de baja laboral por episodio [3], además de ser una de las principales causas de prescripción de antibiótico en nuestro país, hasta en el $80 \%$ de los casos.

En la práctica clínica diaria el diagnóstico se basa en criterios clínicos como fiebre, exudado amigdalar, ausencia de tos y adenopatía cervical anterior dolorosa.

Clásicamente, el tratamiento de las faringoamigdalitis recurrentes ha consistido en antinflamatorios no esteroideos (AINEs), analgésicos, antitusígenos, antibióticos y mucolíticos. El uso excesivo de antibióticos puede producir efectos secundarios en el paciente (intolerancia, alergia), selección de resistencias y el consiguiente aumento del gasto sanitario [4].

Por todo ello, la prevención y el tratamiento adecuado de estas infecciones podrían constituir una modalidad terapéutica prioritaria para prevenir el desarrollo de esta patología.
Cualquier intervención que reduzca la incidencia de infecciones puede tener un gran impacto en la morbilidad y en la calidad de vida de los pacientes, además de incidir directamente en el consumo de recursos sociosanitarios.

El objetivo de este trabajo es revisar las opciones terapéuticas para la prevención y tratamiento de las FAA.

\section{MATERIAL Y MÉTODO}

Se revisaron los criterios clínicos de las FAA, indicaciones de amigdalectomía y pautas de antibioterapia incluidos en las guías clínicas y documentos de consenso de las sociedades científicas europeas.

Se realizó una revisión de otras opciones terapéuticas no contempladas previamente; el empleo de extracto de $P$. Leucotomos, vacunación por la vía de las mucosas, AM3 y betaglucanos, homeopatía y fitoterapia.

Se realizó una búsqueda bibliográfica en las bases de datos ClinicalKey (https://www.clinicalkey.es/) y PubMed (http://www.ncbi.nlm.nih.gov/pubmed) con las palabras clave: Faringitis; Amigdalitis; Amigdalectomía; Enfermedades Otorrinolaringológicas; Terapias complementarias.

Revisión bibliográfica narrativa.

\section{RESULTADOS}

La revisión sistemática de la literatura ofreció un total de 57 artículos, de los cuales, 30 cumplieron los criterios de inclusión. Los resultados deben interpretarse con precaución, pues la calidad metodológica de algunos estudios de inclusión fue variable.

\section{DISCUSIÓN}

La FAA es uno de los principales motivos de consulta en Atención Primaria y en los servicios de urgencias. Es también una de las 
razones más frecuentes por las que se prescribe un antibiótico en nuestro país, con una tasa aproximada de prescripción del 80\% [6]. Los objetivos que se pretenden con el tratamiento son acelerar la resolución de los síntomas, reducir el tiempo de contagio y prevenir las complicaciones. Además se busca otro objetivo a nivel preventivo ya que la FAA representa una causa no despreciable de absentismo laboral.

\section{Definición y concepto de FAA}

\section{Etiología}

La mayoría de las FAA son de causa viral, hasta el $70-80 \%$ de los casos (Tabla 1). Entre los virus, los Adenovirus son los más prevalentes, aunque se ven implicados Enterovirus, Influenza $A$ y $B$, Parainfluenza, VIH, Rinovirus, Coronavirus, Enterovirus, Coxackie $A$, VHS tipo 1 y 2, CMV o VEB [5].

Son las causas bacterianas $y$, en concreto el EBHGA, las que mayores consecuencias clínicas pueden presentar y además llegan a ser responsables de hasta el $30 \%$ de los casos. Es habitual la existencia de portadores asintomáticos de esta bacteria, especialmente en niños. Otras bacterias implicadas en la FAA en nuestro medio son Streptococcus $B$ hemolíticos de los grupos $C$ y $G$. Entre los menos frecuentes encontramos Mycoplasma Neumoniae o Chlamydiophila pneumoniae (Tabla 1).

Tabla 1. Etiología de la faringoamigdalitis aguda

\begin{tabular}{|c|c|c|}
\hline Virus $80 \%$ & Bacterias $10-20 \%$ & Otros \\
\hline Rinovirus & Streptococcus A & $\begin{array}{l}\text { Mycoplasma } \\
\text { neumoniae }\end{array}$ \\
\hline Coronavirus & Streptococcus B & M. Homidis \\
\hline Adenovirus & Streptococcus C & $\begin{array}{l}\text { Chlamydiophila } \\
\text { pneumoniae }\end{array}$ \\
\hline Enterovirus & Anaerobios & Hongos \\
\hline Parainfluenza & $\begin{array}{l}\text { Neisseria Gono- } \\
\text { rrhoeae }\end{array}$ & \\
\hline Influenza A-B & $\begin{array}{l}\text { Corynebacterium } \\
\text { Diphteriae } \\
\text { Y Ulcerans }\end{array}$ & \\
\hline Coxackie A & $\begin{array}{l}\text { Yersinia Enteroco- } \\
\text { lítica }\end{array}$ & \\
\hline $\begin{array}{l}\text { VHS tipo } 1 \mathrm{y} \\
2\end{array}$ & $\begin{array}{l}\text { Arcanobacterium } \\
\text { Haemolyticum }\end{array}$ & \\
\hline VIH & $\begin{array}{l}\text { Treponema } \\
\text { llidum }\end{array}$ & \\
\hline CMV & $\begin{array}{l}\text { Francisella Tula- } \\
\text { rensis }\end{array}$ & \\
\hline VEB & & \\
\hline
\end{tabular}

\section{Epidemiología}

La infección aguda de la mucosa y tejido orofaríngeo constituye una de las causas principales de consultas en Atención Primaria y los servicios de urgencias.

La mayor incidencia se presenta en las estaciones de invierno y primavera, pero existen algunas excepciones. Las FAA ocasionadas por adenovirus son frecuentes durante el verano. Los rinovirus y otros virus producen infecciones de vías altas que predominan durante el final de la primavera o incluso el principio del verano. Por otra parte, las faringitis por Influenza suelen coincidir con fases epidémicas de gripe entre la población, dada su elevada contagiosidad.

La edad del paciente es un elemento importante, aunque la etiología viral es la más frecuente en todos los grupos de edad.

El mecanismo de transmisión suele ser la vía respiratoria a través de las pequeñas gotas de saliva que se expelen al toser, estornudar o simplemente hablar desde una persona infectada a un huésped susceptible [6]. Por el contrario, la transmisión por fómites no parece desempeñar un papel importante en la transmisión de estos microorganismos causantes de la FAA.

En los pacientes adultos, la incidencia de presentación es relativamente baja, si la causa es el EBHGA suele conllevar una baja laboral que puede llegar a ser de hasta los 6 días de absentismo.

Como factores de riesgo van a tener importancia las condiciones de hacinamiento, tabaquismo crónico o contaminación ambiental, pero todos los grupos poblacionales estarán igualmente expuestos independientemente de su condición socioeconómica o profesión.

\section{Clínica}

Como la mayor parte de las FAA son de origen viral la clínica predominante es congestión nasal, febrícula, tos, disfonía, cefalea y mialgias. Típicamente no hay exudados faríngeos ni adenopatías dolorosas y la mejoría de los síntomas se produce en 3-4 días con tratamiento sintomático.

Por el contrario, cuando la etiología es bacteriana, la presentación suele ser un cuadro brusco de fiebre alta con escalofríos, odinofagia y disfagia. Es típico encontrar además leucocitosis, desviación izquierda y reactantes de fase aguda elevados [6] (ASLO, Proteína 
C, Leucocitosis/ml, Neutrofilia, VSG $/ 1^{\text {a }}$ hora) (Tabla 2).

Tabla 2. Diferencias clínicas entre faringoamigdalitis viral y bacteriana.

\begin{tabular}{|l|l|l|}
\hline Caracteristicas & Viral & Bacteriana \\
\hline Edad & $\begin{array}{l}<4 \text { y }>45 \\
\text { años }\end{array}$ & $\begin{array}{l}5-15 \text { años } \\
\text { Invierno y prima- } \\
\text { vera }\end{array}$ \\
$\begin{array}{l}\text { Comienzo } \\
\text { Clínica }\end{array}$ & $\begin{array}{l}\text { Progresivo } \\
\text { Febrícula, } \\
\text { odinofagia } \\
\text { leve }\end{array}$ & Fiebre, odinofagia \\
Otros sínto- \\
mas & $\begin{array}{l}\text { Tos, conges- } \\
\text { tión nasal, } \\
\text { disfonía } \\
\text { Eritema y y } \\
\text { exudado no } \\
\text { purulento }\end{array}$ & $\begin{array}{l}\text { Cefalea, mialgias, } \\
\text { dolor abdominal }\end{array}$ \\
& $\begin{array}{l}\text { Exudado purulen- } \\
\text { to farín- } \\
\text { geo/amigdalar }\end{array}$ \\
\hline
\end{tabular}

\section{Criterios diagnósticos}

El objetivo principal ante una FAA es determinar si su etiología es el EBHGA.

La anamnesis, los datos epidemiológicos y los hallazgos de la exploración física nos orientarán al diagnóstico [7]. Pero los estudios de sensibilidad y especificidad sugieren que el diagnóstico basado sólo en la clínica es erróneo hasta en un $50 \%$ de los casos. Para facilitar el diagnóstico, se han propuesto diversas escalas de puntuación, una de las más empleadas es la de Mclsaac, basada en los criterios de Centor pero ponderando la edad $[8,9]$.

La probabilidad de un resultado positivo en las pruebas de diagnóstico microbiológico es $\leq 3 \%$ en los pacientes que no cumplen ninguno de los criterios clínicos y de un 38-63\% en los que suman 4 o 5 puntos (Tabla 3 ).

Actitud según resultado de los Criterios de Mclsaac:

- 0-1 puntos: no estudio microbiológico (riesgo de infección por EBHGA de un 2 a un $6 \%$ ).

- 2-3 puntos: Estudio microbiológico y tratar sólo si es positivo (riesgo: 10-28\%).

- 4-5 puntos: Estudio microbiológico e iniciar tratamiento antibiótico, si se realiza cultivo, a la espera del resultado (riesgo: $38-63 \%)$.
Tabla 3. Criterios de Mclsaac.

\begin{tabular}{|l|l|}
\hline Criterios & $\begin{array}{l}\text { Pun- } \\
\text { tos }\end{array}$ \\
\hline 1.- Fiebre $\left(>38^{\circ} \mathrm{C}\right)$ & 1 \\
\hline 2.- Hipertrofia o exudado amigdalar & 1 \\
\hline 3.- Adenopatía laterocervical anterior dolorosa & 1 \\
\hline 4.- Ausencia de tos & 1 \\
\hline 5.- Edad & \\
3-14 años & 1 \\
$>15$ años & 0 \\
\hline
\end{tabular}

\section{Diagnóstico diferencial}

Se admite que los tres pilares básicos para el diagnóstico diferencial de las FAA son los criterios clínicos de Centor [8, 10]; el cultivo de exudado faríngeo y la detección rápida en el mismo del antígeno estreptocócico.

Criterios clínicos de Centor [8]:

- Exudado faringoamigdalino.

- Adenopatías cervicales anteriores dolorosas.

- Fiebre.

- Ausencia de tos.

La realización de las pruebas diagnósticas de confirmación de infección estreptocócicas (cultivo y/o test antigénico) debe restringirse a los casos en que se sospeche esta etiología frente al origen viral en base a criterios clínicos. En general se recomienda limitar la administración de tratamiento antibiótico antiestreptocócico a los casos de confirmación etiológica.

Pruebas diagnósticas de confirmación etiológica

Cultivo de exudado faríngeo:

Para la obtención de la muestra, se debe frotar el hisopo contra la pared posterior de la faringe y ambas amígdalas, incidiendo en las zonas que presenten exudado o hiperemia. Debemos emplear un depresor para evitar tocar otras zonas (lengua, úvula,...). El problema es que presenta varias limitaciones $[10,11]$ :

- Se requiere la recogida de la muestra en condiciones adecuadas y su siembra rápida en placas de agar sangre.

- Es preciso que la toma de la muestra se realice antes de la toma de antibiótico. 
- En las mejores condiciones la sensibilidad es del $90 \%$ y su especificidad del 95 al 99\% (Debido a los falsos positivos por ser portadores faríngeos del EBHGA).

- Se precisan de 24 a 48 horas para obtener los resultados del cultivo.

- A pesar esto, el cultivo de exudado faríngeo es la prueba de referencia o patrón oro para establecer o descartar la FAA estreptocócica.

Detección rápida del antígeno estreptocócico: Se realiza a partir del exudado faríngeo y mediante un procedimiento de aglutinación o inmunoabsorción (ELISA) se detecta la presencia de carbohidrato de la pared del estreptococo A. También se puede realizar mediante aglutinación por látex o coaglutinación. El resultado está disponible en 30-60 minutos con una especificidad que alcanza el 95-98\% pero una sensibilidad de puede llegar a oscilar del 50 al $80 \%$ [12]. Un resultado negativo no excluye la infección y obliga a realizar un cultivo faríngeo, además esta prueba tiene como limitación que no es capaz de discriminar entre el estado de portador o infección activa ni es capaz de detectar infecciones por otros estreptococos diferentes del grupo A.

\section{Serología:}

Durante la fase aguda de las faringitis producidas por EBHGA se produce una elevación de los títulos de anticuerpos como ASLO o Ac-antiestreptolisina $O$ [10]. Esta determinación no es útil para para el diagnóstico diferencial pero sí tiene su validez para estudios epidemiológicos o bien para el diagnóstico de complicaciones como la fiebre reumática o glomerulonefritis postestreptocócica.

\section{Opciones terapéuticas}

\section{Amigdalectomía}

El documento de consenso entre la Sociedad Española de Otorrinolaringología y Patología Cervicofacial y la Asociación Española de Pediatría realizaron una actualización de las indicaciones de amigdalectomía [2]. En dicho consenso se encuentran las indicaciones y recomendaciones para la amigdalectomía en procesos infecciosos.

\section{Amigdalitis de repetición}

Se definen como amigdalitis de repetición o amigdalitis recurrentes las siguientes situaciones clínicas

- 7 o más episodios de amigdalitis aguda al año en el último año o,

- 5 episodios al año en los últimos dos años o,

- 3 episodios al año en los últimos 3 años.

- Síntomas persistentes durante al menos 1 año.

Además, cada episodio debe cumplir, al menos, uno de los siguientes criterios:

- Exudado purulento sobre las amígdalas.

- Fiebre superior a $38^{\circ} \mathrm{C}$.

- Linfadenopatías cervicales anteriores.

- Cultivo faríngeo positivo para EBHGA.

Estos son los criterios denominados mínimamente aceptables. No obstante, cada caso debe ser evaluado en particular sopesando los siguientes factores (en este caso aplicado a la población infantil):

- Los episodios de amigdalitis son incapacitantes e impiden el desarrollo normal de las actividades del niño.

- Tratamiento adecuado en cada episodio.

- Los episodios de amigdalitis trastornan la vida familiar y laboral de los padres.

- La curva de crecimiento del niño se estanca sin otra razón que lo explique.

- Los episodios de amigdalitis deberían estar documentados en la historia clínica del paciente. Si no es así, y la historia clínica ofrece dudas, se procederá al seguimiento del paciente durante 6 meses para confirmar el patrón clínico y poder considerar la indicación de operación.

\section{Absceso periamigdalino recurrente.}

Se considera indicación quirúrgica la presentación de dos casos consecutivos de absceso periamigdalino [13].

\section{Adenitis cervical recurrente.}

Se define como adenitis cervical el siguiente cuadro clínico:

- Inflamación aguda de adenopatías cervicales múltiples.

- Fiebre superior a $38^{\circ}$ y malestar general

- Más de 3 días de duración.

- Ausencia de infección respiratoria baja.

- Coexistencia de infección respiratoria alta o amigdalitis aguda. 
Se define como adenitis cervical recurrente la repetición de este cuadro clínico con la misma frecuencia considerada para la amigdalitis recurrente.

Las consideraciones a la hora de evaluar estos casos son las mismas que las descritas para la amigdalitis recurrente.

\section{Antibioterapia}

Según las guías actuales, el antibiótico de elección es la penicilina, porque tiene el espectro de acción más estrecho, es más económica y no se ha documentado ningún aislamiento de EBHGA resistente a la misma [9]. Si se han descrito cepas tolerantes in vitro en las que el efecto del antibiótico ha sido exclusivamente bacterioestático [8], no obstante in vivo, no se han demostrado diferencias significativas en los índices de fracaso terapéutico entre cepas tolerantes y susceptibles.

Debe administrarse el tratamiento antibacteriano durante al menos 8 días, aunque preferentemente se recomienda administrarlo durante 10 días.

Penicilina:

- Fenoximetilpenicilina potásica o penicilina V (1.200.000 Ul/12h por vía oral), la Fenoximetilpenicilina benzatina está disponible en nuestro país en suspensión oral, fácil de administrar y con mejor sabor que la potásica.

- La penicilina $G$ benzatina intramuscular en dosis única fue de elección hace años, pero el importante dolor en la zona de la inyección hizo que se sustituyera por la penicilina $\mathrm{V}$ oral que demostró igual eficacia sin desarrollo de resistencias. Sin embargo, precisa un tratamiento más prolongado de difícil cumplimiento cuando se nota mejoría de los síntomas en los primeros 3 o 4 días.

Tratamiento antibiótico de la FAA estreptocócica $[6,5]$.

Primera elección:

- Penicilina V (Fenoximetilpenicilina potásica o Benzatina) durante 10 días.

- Menores de 12 años y de $27 \mathrm{Kg}$ : $250 \mathrm{mg}$ cada $12 \mathrm{~h}$.

- Mayores de 12 años o de $27 \mathrm{Kg}$ : $500 \mathrm{mg}$ cada $12 \mathrm{~h}$.

- Amoxicilina durante 10 días. 40$50 \mathrm{mg} / \mathrm{Kg} /$ día cada 12 o 24h. Máximo $500 \mathrm{mg}$ cada $12 \mathrm{~h}$ o $1 \mathrm{gr}$ cada $24 \mathrm{~h}$.
En caso de mal cumplimiento vía oral o vómitos:

- Penicilina $\mathrm{G}$ benzatina, dosis única IM profunda:

- Menores de 12 años y s25Kg: 600.000 UI im.

- Mayores de 12 años y $\geq 25 \mathrm{Kg}$ : 1.200.000 UI im.

En caso de alergia a Penicilina:

- Reacción retardada: Cefadroxilo $30 \mathrm{mg} / \mathrm{Kg} /$ día cada $12 \mathrm{~h}$. Máximo $1 \mathrm{gr}$ cada $24 \mathrm{~h}$.

- Reacción inmediata: Azitromicina:

- Menores de 12 años: 10-12mg/Kg/día, en una dosis durante 3-5 días.

- Mayores de 12 años: 500mg diarios el primer día seguidos de $250 \mathrm{mg}$ en una única dosis hasta 5 días.

Si resistencia a Macrólidos:

- Clindamicina:20-30mg/Kg/día cada 8-12 horas, 10 días (máximo 900mg/día).

- Josamicina:30-50mg/Kg/día, cada $12 \mathrm{~h}$, 10 días (máximo 1gr/día).

- Diacetato de midecamicina: $40 \mathrm{mg} / \mathrm{Kg} /$ día, cada 12 h, 10 días (máximo $1,5 \mathrm{gr} /$ día).

\section{Otros tratamientos}

\section{Extracto de P. Leucotomos}

El extracto de Polypodium Leucotomos es un fármaco inmunomodulador de administración oral utilizado habitualmente en dermatología y en patologías cuya etiología está basada en un déficit de linfocitos $T$ supresores, ya que reduce la producción de células proliferativas, aumenta el número de células p53(+) y aumenta la capacidad antioxidante plasmática [14].

En la literatura encontramos dos estudios realizados en pacientes con faringoamigdalitis de repetición. Cardelús y cols. (2011), estudio prospectivo y aleatorizado con un tamaño muestral de 49 pacientes [15]. En él se concluye que la capacidad moduladora de la respuesta inmune e inflamatoria de $P$. Leucotomos permite suponer que la utilización de este fármaco sería beneficiosa en el control de las faringoamigdalitis recidivantes. Así mismo refiere que sería necesario una segunda fase del estudio para seguimiento a 
largo plazo con un mayor número de casos para poder comprobar este efecto. También indican que la valoración de la mejoría clínica se ha registrado por datos subjetivos (presencia o ausencia de disfagia, odinofagia, fiebre, tos, parestesias, artralgias y astenia) que refería el paciente, aunque objetivan esos datos al correlacionarse con una disminución de inmunoglobulinas ( $\lg A$, IgG e IgM sin realizar distinción) y disminución de la titulación de ASLO.

El otro estudio revisado, de Espuch Nuñez y cols. [16] consta de 63 pacientes. Se trata de un estudio prospectivo y observacional no aleatorizado. Al igual que en el estudio de Cardelús se incluyeron como parámetros objetivos de la mejoría clínica (aunque no especifican que escala se empleó para la gradación de los síntomas), la titulación de ASLO, IgG, IgM e IgA así como FR. Además de la mejoría clínica subjetiva referida por los pacientes el estudio registró aumentos no significativos de $\lg \mathrm{M}$ e $\lg \mathrm{A}$ y significativo de IgG. La titulación de ASLO se encontraba elevada en el $24 \%$ de los pacientes.

En ambos estudios, uno de los pilares en los que se basan para indicar el extracto de $P$. Leucotomos como coadyuvante al tratamiento de las FAA es la ausencia de efectos adversos detectados y ausencia de complicaciones.

\section{Vacunación por la vía de las mucosas}

El tratamiento de inmunoterapia con nuevos inmunógenos bacterianos está emergiendo como una alternativa terapéutica capaz de estimular la inmunidad innata y la adaptativa específica de sujetos con diversas patologías. El concepto sistema inmunitario de las mucosas (también conocido como MALT de sus siglas en inglés The Mucosa-Associated Lymphoid Tissue) hace referencia a la función inmunitaria que comparten las mucosas de vías respiratorias, tracto digestivo, glándulas lagrimales, ojo, oído, mama y aparato genitourinario. Sus más de $400 \mathrm{~m}^{2}$ de superficie lo convierten en el más extenso del organismo. Sus funciones principales, son proteger y prevenir contra la invasión y colonización del organismo por microrganismos patógenos, a la par que reconocer y captar alérgenos y antígenos extraños. También modulan el desarrollo de las respuestas inmunológicas e inflamatorias cuando un agente extraño penetra en el organismo.
La administración sublingual se ha venido utilizando básicamente para aplicar vacunas de mucosas antialérgicas. La vacunación, consiste en una estimulación inmune específica activa. Las vacunas, inducen específicamente la producción de linfocitos $\mathrm{T}$ de memoria y linfocitos $B$, favoreciendo el desarrollo de una respuesta eficaz si se produce una infección por ese patógeno.

Bergeman y cols. [17] realizaron el primer metanálisis que analizó la eficacia de un lisado bacteriano frente a placebo en pacientes que presentaban bronquitis crónica en la reducción de exacerbaciones agudas. También llevaron a cabo un análisis de la relación coste/efectividad.

La mayoría de los estudios están realizados en pacientes con diagnóstico de EPOC (Enfermedad Pulmonar Obstructiva crónica, COPD de sus siglas en inglés Chronic Obstructive Pulmonary Disease). Steurer-Stey y cols.[18] llevaron a cabo una revisión sistemática de los resultados de 13 ensayos clínicos aleatorizados y controlados con placebo. En total se incluyeron 1.971 pacientes, pero sólo 731 pacientes (que correspondían a dos estudios) tenían según estos autores una metodología apropiada y comunicaban que acción se producía sobre las exacerbaciones.

Una serie de estudios clínicos no controlados sobre infecciones respiratorias recurrente en adultos y niños han mostrado que la administración oral de lisados bacterianos polivalentes mejoran [19]. la respuesta clínica reduciendo el número, duración y la gravedad de los episodios infecciosos.

Uno de los estudios llevados a cabo por Alecsandru y cols. en 2011 evaluó el efecto de la administración de una vacuna de mucosas por vía sublingual diaria en una cohorte de pacientes 17 pacientes adultos en la prevención de las infecciones respiratorias [20]. Al inicio y final del tratamiento de inmunización se llevaron a cabo determinaciones inmunológicas séricas y se valoró el impacto en el control clínico basándose en la incidencia de las infecciones de vías respiratorias superiores e inferiores. Se compararon con los episodios que cada paciente había presentado en los 12 meses previos al tratamiento. Este estudio clínico piloto sugirió que se produce un efecto beneficioso en la respuesta clínica de las infecciones de vías respiratorias asociado a una activación de la respuesta $T$ CD4+ linfoproliferativa frente a los antígenos bacterianos. 
En conjunto estos datos son consistentes con otros estudios que demuestran una asociación entre la inmunización con inmunomoduladores bacterianos polivalentes y la mejoría clínica de enfermedades respiratorias. En la mayoría de los estudios, los resultados valoran las incidencia/recurrencia de infecciones de vías respiratorias, especialmente como indicamos previamente en pacientes con EPOC. No hay datos que valoren específicamente la Faringoamigdalitis recurrente del adulto.

III. Am3 y betaglucanos procedentes de Pleurotus Ostreatus y Vitamina $C$

La revision realizada en la literaura no muestra ningún estudio del empleo de AM3 (glicopéptido adsorbido en fosfato cálcico) en pacientes que presenten Faringoamigdalitis agudas, recurrentes. Por el contrario sí se encuentran estudios en pacientes con EPOC (Enfermedad Pulmonar Obstructiva Crónica) y otras patologías del área ORL como OMS (Otitis Media Serosa) o Aftosis recurrente.

AM3 es un fármaco inmunomodulador de administración oral [21, 22]. Presenta capacidad adyuvante frente a infecciones víricas y bacterianas, a la par que produce una recuperación funcional de células NK, monocitos, polimorfonucleares y linfocitos T. Además, posee efecto antiinflamatorio al producir una inhibición parcial sobre la liberación de diferentes citoquinas.

La mayoría de estudios sobre el empleo de Betaglucanos de "Pleurotus ostreatus» y Vitamina $C$ en la prevención de infecciones respiratorias recurrentes se han llevado a cabo en población pediátrica [23, 24] . Encontramos algunas publicaciones con su empleo como tratamiento complementario y alternativo en la prevención del «resfriado común». Existe una revisión llevada a cabo por Nahas en 2011 [25]. sobre las publicaciones Ilevadas a cabo entre 1996 y 2011 donde se incluyeron estudios prospectivos y ensayos clínicos revisados de las bases de datos Medline, Embase y Cochranes Database. En él se concluye que la Vitamina $\mathrm{C}$ se puede recomendar en población canadiense para la prevención del resfriado común, aunque no indica el nivel de evidencia.

Los resultados de los estudios en población pediátrica indican que en ausencia de medidas para la inmunización activa contra los patógenos más frecuentemente asociados a procesos infecciosos de las vías respiratorias, los resultados sugieren que la suplementación profiláctica con betaglucanos procedentes de $P$. Ostreatus y Vitamina $\mathrm{C}$ podría ser beneficiosa, particularmente en niños con patrón recurrente y factores de riesgo específicos. Concretamente, el estudio de Sapena 2015 [23]. consideró como procesos infecciosos (otitis, resfriados comunes, faringoamigdalitis, laringitis y bronquitis) por lo que no se valoran específicamente los cuadros de FAA.

\section{Homeopatía}

Se entiende por terapia homeopática una alternativa al tratamiento convencional cuando aún no se ha llegado a un estadio de infección grave [26]. Se entiende como una terapia de estímulo y regulación del propio cuerpo para conseguir el resultado terapéutico. En este sentido, el punto de mira no se fija en la función que sufre el trastorno, sino en la persona, con sus particularidades y capacidades para tratar y defenderse de las enfermedades.

Los medicamentos homeopáticos se utilizan tanto en las enfermedades agudas como en las crónicas. En la literatura encontramos el estudio Baranow [27] realizado entre los años 1995 y 1996. Se realizó un estudio prospectivo y multicéntrico para valorar la eficacia y tolerancia de un preparado combinado (Atropinum sulfuricum, Mercurius bijodatus, Herpar sulfuris, Kalium bichhromicum y Silicea). En él, participaron 193 consultas médicas generales, de pediatría y ORL en Alemania, Rusia y Ucrania. En total, formaron parte del estudio 1368 pacientes de ambos sexos y edades entre los 6 y 35 años, con una duración de tratamiento de 14 días. El estudio indica que se les permitió emplear medidas adicionales durante el tratamiento, esto incluía «protección de garganta», «inhalaciones» o "gárgaras». No se distingue aquellos pacientes que emplearon estas medidas de los que no las requirieron ni el principio activo que las componía. En estos pacientes se realizaron exámenes de control al segundo, sexto y decimocuarto días. En aquellos pacientes que padecían faringoamigdalitis recurrente el estudio se prolongó 6 semanas más como profilaxis de la recidiva. En estos casos, se llevaba a cabo una cuarta visita (que coincidía con la octava semana de tratamiento). En ellos se valoró la eficacia y tolerancia del preparado para la amigdalitis aguda y reagudizaciones de amigdalitis crónica recurrente. El estudio 
también incluyó pacientes amigdalectomizados. La evaluación del resultado de la terapia se realizó por parte del médico examinador (mediante una estimación de la tolerancia al tratamiento) y del paciente empleando una escala dividida en 5 puntos. La escala incluía «sin molestias», «mejorado notablemente», «mejorado», «invariable», «empeorado», además valoraban el sabor del preparado. Para la mayoría de los parámetros de los síntomas típicos de la amigdalitis se llegó a una remisión del $80-90 \%$ siendo buena la tolerancia. El estudio indica que los pacientes que presentaron éxito medicamentoso no requirieron tratamiento antibiótico durante el estudio pero no se habla de recaídas ni de profilaxis.

Este estudio es el que presenta mayor tamaño muestral, pero ya desde 1954 se habían realizado exámenes clínicos con buenos resultados para el tratamiento de amigdalitis aguda y crónica, pero no cumplían los criterios que requiere un estudio clínico.

Este estudio incluye juntos pacientes de distintos grupos etarios, pacientes pediátricos desde los 6 años de edad y un grupo de adultos que no superan los 35 años. Esto hace que sea difícil comparar los resultados con otros estudios que han seguido diferente metodología. Igualmente ocurre al haber incluido pacientes amigdalectomizados sin diferenciar amigdalitis de faringitis o faringoamigdalitis. Tampoco indican los criterios diagnósticos que siguieron para considerar las patologías y no especifican si los cuadros eran virales o bacterianos.

\section{Fitoterapia}

Los productos farmacéuticos y medicinales basados en la fitoterapia se han empleado clásicamente en el tratamiento de las FAA.

El artículo publicado por Ciuman [28] en 2012 hace una revisión del empleo de la fitoterapia y terapias naturales en la Comunidad Europea para el tratamiento de patologías otorrinolaringológicas. La revisión se basa en estudios clínicos y metanálisis.

En él se revisan tratamientos empleados en faringoamigdalitis agudas y cuadros recurrentes. Se emplean distintas presentaciones; soluciones para realizar gargarismos y preparados comercializados en forma de grageas, gotas para administración oral e incluso comprimidos.

En todo momento, el artículo hace referencia a un uso como tratamiento adyuvante o tera- pia simultánea con otros medicamentos. Refiere que de forma general no se usan como tratamiento de patología aguda sino en situaciones clínicas no graves y sobretodo en enfermedades crónicas. Hace una revisión por patologías y síntomas clínicos (tos, otitis media, faringolaringitis aguda y crónica...) y no se hace referencia a resultados estadísticos ni forma de recogida de datos de los estudios revisados.

Para el tratamiento de las FAA se emplean diferentes combinaciones de hojas de Salvia officinalis, Thymus vulgaris, Matricaria recutita, Althaea officinalis, Quercus robur, Juglans regia, Achilea Millefolium, Equisetum arvense, Taraxacum officinale.

Dos importantes fitoterapéuticos [25] con propiedades antimicrobianas son Tropaeolum majus y Armoracia rusticana que al contener aceite de mostaza tienen propiedades antibacterianas frente a cepas grampositivas $y$ gramnegativas. También presentan propiedades virostáticas y efectos antimicóticos por lo que se podrían emplear para evitar el uso de antibióticos.

La combinación de dos hierbas medicinales, Nasturtium officinale y raíz de rábano picante comercializadas en forma de comprimidos permite emplear los efectos sinérgicos de ambas plantas [29]. En particular, es efectiva frente a Pseudomonas con una eficacia comparable a la antibioterapia según el estudio de Conrad y cols. de un estudio in vitro.

Como tratamiento sintomático de las FAA la corteza de sauce (Salix purpurea, S.daphnoides, S.fragilis) pueden disminuir la temperatura corporal. La principal diferencia con respecto al ácido acetilsalicílico (AAS) es que la salicilina caree de grupo acetilo y por tanto los efectos secundarios a nivel gástrico y de agregación plaquetaria son menores [30].

Otros preparados con efecto antipirético contienen Sambucus nigra, Tilia platyphyllos, Tilia cordata y Tilia vulgaris.

Con frecuencia se emplea para el tratamiento de la amigdalitis aguda y crónica la asociación de Calendula officinalis y Pelargonium sidoides, debido a su efecto antibacteriano, antiviral y mucolítico [31]. En este caso, el uso está fuera indicación ya que a pesar de su empleo no hay suficientes datos clínicos que lo avalen [28].

La revisión de los estudios resulta dificultosa debido a los distintos criterios de inclusión de pacientes. No se distinguen grupos de edad e 
incluso en alguno de ellos se incluye población pediátrica. Otro elemento que dificulta la comparativa es la diferencia de tamaños muestrales entre los estudios y la inclusión de pacientes amigdalectomizados. Muchos de ellos incluyen seguimiento a corto plazo, y no aclaran el criterio diagnóstico de faringoamigdalitis recurrente. Algunos de los estudios realizados consideraban el empleo simultáneo de diversas opciones terapéuticas sin realizar distinciones entre grupos.

Uno de los problemas más recurrentes es que no se distinguen claramente si los procesos son de etiología viral o bacteriana.

Ninguno de ellos incluye como prueba diagnóstica el cultivo de exudado ni la detección rápida del antígeno. Por otra parte es completamente comprensible, ya que encarecería mucho un estudio cuya base es el ahorro del gasto sanitario. Pero debemos ser conscientes a la hora de revisar un estudio que hasta en un $50 \%$ de los casos el diagnóstico basado en la clínica es erróneo [7].

Otra variable a revisar es la escasa uniformidad en las escalas que valoran las variaciones clínicas que experimentan los pacientes con los diferentes tratamientos. Como opciones para resolver esta disparidad se puede emplear algún cuestionario de de salud como el Euroqol-5D-5L [32]. Se trata de un instrumento genérico y estandarizado elaborado para describir y valorar la calidad de vida relacionada con la salud (CVRS). Es genérico, porque no hace referencia a ninguna enfermedad específica. Está estandarizado porque con este instrumento se pretende valorar un conjunto estandarizado de estados de salud. EI EQ-5D ha probado su utilidad como medida de salud de la población, pudiendo mostrar las diferencias entre comunidades o grupos de población de diferentes características socioeconómicas, tanto de los estados de salud como de la valoración que los individuos hacen de esos estados de salud.

\section{CONCLUSIONES}

- La etiología viral es la más frecuente en la faringoamigdalitis en todos los grupos de edad.

- El Estreptococo beta Hemolítico Grupo A es responsable de hasta el $30 \%$ de los casos.

- Los tres pilares básicos para el diagnóstico diferencial de las FAA son: Los crite- rios clínicos de Centor, el cultivo de exudado faríngeo y la detección rápida en el mismo del antígeno estreptocócico.

- El tratamiento de la faringoamigdalitis recurrente del adulto incluye la amigdalectomía, antibioterapia y otros tratamientos como, extracto de $P$. Leucotomos, Vacunación por la vía de las mucosas, AM3, Homeopatía o Fitoterapia.

- Los estudios revisados que valoran el empleo de otros tratamientos en la faringoamigdalitis recurrente del adulto son metodológicamente muy diferentes. Por lo tanto, los resultados no son comparables.

- Serían necesarios estudios bien diseñados que comparen las distintas opciones terapéuticas de la faringoamigdalitis recurrentes.

\section{BIBLIOGRAFÍA}

1. Cenjor C, García-Rodríguez JA, Ramos $A$, Cervera J, Tomás $M$, Asensi $F$, et al. Documento de consenso sobre tratamiento antimicrobiano de la faringoamigdalitis. Acta Otorrinolaringol Esp 2003;54:369-83.

2. Cervera Escario J, del Castillo Martin F, Gómez Campdera JA, Gras Albert JR, Pérez Piñeiro B, Villafruela Sanz MA. Indicaciones de Adenoidectomía y Amigdalectomía: Documento de Consenso entre la Sociedad Española de Otorrinolaringología y Patología Cervicofacial y la Asociación Española de Pediatría. Acta Otorrinolaringol Esp 2006;57:59-65.

3. Herranz Jordán B, Pérez Martín C. Encuesta sobre prescripción de antibióticos en infecciones respiratorias pediátricas. Rev Pediatr Aten Primaria 2005;7:55778.

4. World Health Organization. WHO's first global report on antibiotic resistance reveals serious, worldwide threat to public health. Antimicrobial resistance-global surveillance report. Virtual Press Conference. 30 april 2014. Disponible en: http://www.euro.who.int/en/healthtopics/disease-prevention/antimicrobialresistance/news/news/2014/04/newreport-antibiotic-resistance-a-globalhealth-threat. 
5. Costa Cerdá A, Martínez Lasheras MB, Torralba González de Suso M, Rodríguez Zapata M. Protocolo diagnóstico y tratamiento empírico de la faringoamigdalitis. Medicine 2010;10(56):3873-7.

6. Cots JM, Alós JI, Bárcena M, Boleda X, Cañada JL, Gómez N, et al. Recommendations for management of acute pharyngitis in adults. Acta Otorrinolaryngol Esp 2015;66(3):159-70.

7. Madurell J, Balaqué M, Gómez M, Cots JM, Llor C. Impact of rapid antigen detection testing on antibiotic prescription in acute pharyngitis in adults. FARINGOCAT Study: a multicentric randomized controlled trial. BMC Farm Pract 2010;11:25.

8. Centor RM. When Should Patients Seek Care for Sore Throat? Ann Intern Med. 2013;159(9):636-7.

9. Mclsaac WJ, Goel V, To T, Low DE. The validity of a sore throat score in family practice. CMAJ. 2000;163(7):811-5.

10. Sanz Moreno J. Protocolo de diagnóstico diferencial de las faringoamigdalitis. Medicine 2010;10(58):4015-8.

11. Piñeiro $R$, Hijano $F$, Álvez $F$, Fernández A, Silva JC, Pérez C, et al. Documento de consenso sobre el diagnóstico y tratamiento de la faringoamigdalitis aguda. An Pediatr 2011 Nov;75(5):342.e1-13.

12. Bisno AL, Gerber MA, Gwaltney JM, Kaplan EL, Schwartz RH. Practice guidelines for the diagnosis and management of group A streptococcal pharyngitis. Clin Infect Dis 2002;35:113-25.

13. Villafruela Sanz MA. Indicaciones de adenoidectomía, amigdalectomía y tubos de timpanostomía. En AEPap ed. Curso de Actualización Pediatría 2012. Madrid: Exlibris Ediciones; 2012. pp. 39-44.

14. Rodriguez-Yanes E, Juarranz Á, Cuevas J, González S, Mallol J. Polypodium leucotomos decreases UV-induced epidermal cell proliferation and enhances p53 expression and plasma antioxidant capacity in hairless mice. Exp Dermatol 2012;21(8):638-40.
15. Cardelús $S$, Cardesín A, Martínez A, Escamilla $Y$, Rosell $R$, et al. Uso de anapsos en el tratamiento de la faringoamigdalitis crónica recidivante. ORL Aragón. 2011;14(2):25-7.

16. Espuch Nuñez D, Martín del Guayo G. Faringoamigdalitis crónica en niños. Respuesta con anapsos. ORL Aragón. 2014;17(1):6-9.

17. Bergemann $R$, Brandt $A$, Zoellner $U$, Donner CF. Preventive treatment of chronic bronchitis: a meta-analysis of clinical trials with a bacterial extract (OM$85 \mathrm{BV}$ ) and a cost-effectiveness analysis. Moraldi Arch Chest Dis 1994;49:302-7.

18. Steurer-Stey C, Bachmann LM, Sterurer J, Tramber MR. Oral purified barcterial extracts in chronic bronchitis and COPD: Systematic review. Chest 2004;126:1645-55.

19. Bellanti JA, Settipane RA. Bacterial vaccines and the inmune system: a journey of rediscovery for the allergistinmunologist and all health care providers. Allergy Asthma Proc 2009;30:S3-4

20. Alecsandru D, Valor L, Sánchez-Ramón S, Gil J, Carbone J, Navarro J, et al. Sublingual therapeutic immunization with a polyvalent bacterial preparation in patients with recurrent respiratory infections: inmunomodulatory effect on antigen-specific memory CD4+ $T$ cells and impact on clinical outcome. Clin Exp Inmunol 2011;164(1):100-7.

21. Prieto A, Reyes E, Bernstein ED, Martinez B, Monserrat J, Izquierdo JL, et al. Defective natural Killer and phagocytic activities in chronic obstructive pulmonary disease are restored by glycophosphopeptical (inmunoferon). Am J Respir Crit Care Med 2001;163:1578-83.

22. Ortega del Álamo P, Rivera Rodríguez T, Sanz Fernández R. Efecto de AM3 sobre la resolución de la otitis media serosa (OMS) en pacientes pediátricos. Acta otorrinolaringol Esp 2005;56:1-5.

23. Sapena Grau J, Picó Sirvent L, Morera Inglés M, Rivero Urgell M. Betaglucanos de "Pleurotus ostreatus" en la prevención de infecciones respiratorias recurrentes. Acta Pediatr Esp 2015;73(8):186-93. 
24. Jesenak M, Majtan J, Rennerova Z, Kyselovic J, Banovcin P, Hrubisko $M$ Immunomodulatory effect of pleuran $(\beta$ glucan from Pleurotus ostreatus) in children with recurrent respiratory tract infections. International Immunopharmacology. 2013;15:395-9.

25. Nahas R, Balla A. Complementary and alternative medicine for prevention and treatment of the common cold. Can Fam Physician 2011;57(1):31-6.

26. Friese $\mathrm{KH}$. Alternative treatment methods in ENT. HNO 1997;45(8):593-607.

27. Baranow A, Moschitsch P, Friese K, Heger M. Alternative Behandlung der Angina tonsillaris, Ergebnisse einer offenen multinationalen Beobachtungsstudie unter Berücksichtigung de OutcomesForschungsansatzes,

Kassenarzt 2000;11:40-9.

28. Ciuman RR. Phytotherapeutic and naturopathic adjuvant therapies in otorhinolayngology. Eur Arch Otorhinolaryngol 2012;269:389-97.
29. Conrad A, Kolberg T, Engels I, Frank U. In vitro study to evaluate the antibacterial activity of a combination of the haulm of nasturtium (Tropaeoli majoris herba) and of the roots of horseradish (Armoriaceae rusticanae radix). Arzneimittelforschung 2006; 56(12):842-49.

30. März RW, Kemper F. Willow bark extracteffects and effectiveness. Status of current knowledge regarding pharmacology, toxicology and clinical aspects. Wien Med Wochenschr 2002;152:354-9.

31. Conrad A, Kolodziej H, Schulz V. Pelargonium sidoidextract (EPs 7630): registration confirms efficacy and safety. Wien Med Wochenschr 2007;157:331-6.

32. Ministerio de Sanidad, Servicios Sociales e Igualdad. Encuesta Nacional de Salud. España 2011/12. Calidad de vida relacionada con la salud en adultos: EQ-5D-5L. Serie Informes monográficos n. ${ }^{\circ}$ 3. Madrid: Ministerio de Sanidad, Servicios Sociales e Igualdad, 2014. Disponible en: http://www.msssi.gob.es 